\title{
Modulation of novel DNA adducts during human uterine cervix cancer progression
}

\author{
SRIVANI RAVOORI ${ }^{1}$, MANICKA V. VADHANAM ${ }^{1}$, DIANE D. DAVEY ${ }^{3}$, \\ CIDAMBI SRINIVASAN $^{4}$, BALA NAGARAJAN ${ }^{5}$ and RAMESH C. GUPTA ${ }^{1,2}$ \\ ${ }^{1}$ James Graham Brown Cancer Center, ${ }^{2}$ Department of Pharmacology and Toxicology, University of Louisville, \\ Louisville, KY 40202; Departments of ${ }^{3}$ Pathology and Laboratory Medicine, ${ }^{4}$ Statistics, \\ University of Kentucky, Lexington, KY 40506, USA; ${ }^{5}$ Department of Microbiology \\ and Tumor Biochemistry, Cancer Institute, Chennai 600 020, India
}

Received May 2, 2006; Accepted June 16, 2006

\begin{abstract}
Progressive accumulation of DNA lesions leads to genetic mutations that are central to the process of tumorigenesis. Human cervix provides an ideal system to determine progressive accumulation of DNA adducts in the target tissue because of its accessibility during routine diagnostic checkups. Uterine cervix samples from various pathologies, i.e. normal $(n=13)$, inflammation $(n=9)$, dysplasia $(n=5)$ and different stages of invasive cancer $(n=47)$, were analyzed for DNA adduct burden by modified ${ }^{32} \mathrm{P}$-postlabeling/TLC systems. Six subgroups of adducts were detected in the following descending order of polarities: P-1, P-2, PL-1, PL-2, L-1 and L-2 (P, polar; L, lipophilic; PL, between polar and lipophilic). No qualitative differences were observed in adduct profiles in the various cervix pathologies analyzed. However, significant quantitative differences were found. Previously known lipophilic adducts increased significantly from normal to cancer ( $144 \pm 61$ to $503 \pm 51$ adducts $/ 10^{9}$ nucleotides). Interestingly, the newly discovered polar adducts were present at 61- to 527-fold higher levels than lipophilic adducts. Of all the polar adducts, the known mutagenic lesion, 8-oxodeoxyguanosine, predominated in all cervix conditions. Notably, this lesion was elevated 27 -fold in inflammation compared with normal cervix $\left(51,058 \pm 9,863\right.$ versus $1,886 \pm 507$ adducts $/ 10^{9}$ nucleotides $)$. The P-1, PL-1, PL-2 and L-1 adducts were elevated 3- to 13-fold in inflammation compared with normal cervix, and were also higher in dysplasia and cancer. Our data suggest that inflam-
\end{abstract}

Correspondence to: Dr Ramesh Gupta, James Graham Brown Cancer Center and Department of Pharmacology and Toxicology, 304E Delia Baxter Research Building, 580 S. Preston St., University of Louisville, Louisville, KY 40202, USA

E-mail:rcgupta@louisville.edu

Abbreviations: 8-OxodG, 8-oxodeoxyguanosine; CIN, cervical intraepithelial neoplasia; DRZ, diagonal radioactive zone; HPV, human papillomavirus

Key words: DNA adducts, oxidative DNA damage, ${ }^{32} \mathrm{P}-$ postlabeling, cervical cancer, inflammation mation may be involved in directing the course of disease progression by accumulating higher levels of DNA lesions. The data further suggest the biomarker potential of the newly detected array of DNA adducts.

\section{Introduction}

During the normal metabolic process, human cells are exposed to endogenous, DNA-reactive substances such as hydroxyl radicals, and lipid oxidation products such as malondialdehyde, and $\alpha, \beta$-unsaturated aldehydes (enals) (reviewed in ref. 1). Nitric oxide, a free radical produced by phagocytes, and by the induction of NO synthase by inflammatory cytokines (2), undergoes mutual reaction to produce peroxynitrite, which also generates hydroxyl radicals that oxidize or deaminate DNA (3). Hydroxyl radicals can produce over 20 oxidized DNA bases (4). Endogenous DNA adducts have been estimated at $>10,000$ per $10^{9}$ nucleotides (5). 8-Oxodeoxyguanosine (8OxodG), thymidine glycol and 5-hydroxymethyl-dU are the commonly observed lesions in mammalian DNA. 8-OxodG is highly mutagenic, producing $\mathrm{G} \rightarrow \mathrm{T}$ transversions, while thymidine glycol ( $\mathrm{T} \rightarrow \mathrm{C}$ transitions) and 5-hydroxymethyl-dU $(\mathrm{C} \rightarrow \mathrm{T}$ transitions) are weakly mutagenic (6). Other adducts from reactive intermediates include cyclic adducts that are formed from lipid peroxidation products. Cyclic adducts also induce transversion and transition mutations, depending upon the type of adducts (7-9).

The novel ${ }^{32} \mathrm{P}$-postlabeling/TLC systems, developed recently in our laboratory, have been employed to measure collectively a host of DNA adducts encompassing high polarity to high lipophilicity (Gupta RC et al, manuscript in preparation). This technology, termed 'adductomics' allows global detection of DNA adducts. We set out to test this new technology in diseases that have clearly defined stages of progression, and the target tissue is easily accessible. Human uterine cervical lesions progress from low-grade to highgrade intra-epithelial lesions to invasive carcinoma. Procurement of the samples by non-invasive methods during routine annual checkups, and the ability of the Pap smear to detect the progression of the disease make the cervix one of the best models to study the process of human carcinogenesis 
and evaluate the biomarker potential of newly developed tech-nologies besides diagnostic and therapeutic interventions (10).

Cancer of the cervix uteri is the second most common form of cancer in women worldwide, with low- and mediumresource countries contributing to approximately $80 \%$ of the burden. In the United States, approximately 9,710 new cases are expected to be diagnosed and approximately 3,700 women are expected to die due to this cancer in 2006 (11). The incidence rate of invasive cervical cancer in Kentucky is $40 \%$ higher than the SEER rate during 1991-1998 (12); it ranks fifth in incidence with age-adjusted rate $(11.1 / 100,000)$, which is higher than the national average $(8.4 / 100,000)$. Epidemiological studies confirm a causal role of human papillomaviruses (HPV) infection in cervical carcinogenesis. However, while $>99 \%$ of the invasive cancers are HPVpositive (13), it is important to acknowledge that only $<5 \%$ of all HPV-positive cases progress to develop invasive cancer (14). Therefore, attention has been given to several co-factors that influence the outcome of a cervix infection, including tobacco smoking, oral contraceptive use and parity (15). In addition, an association was found between cervical inflammation and high-grade lesions in women infected with HPV (16). Elevated levels of inflammatory cytokines IL-6 and IL-8 in cervicovaginal secretions (17) and in cervical cancers $(18,19)$ have been reported. In this study, we examined the influence of cervical inflammation and other forms of cervix pathogenesis on the accumulation of DNA adducts.

\section{Materials and methods}

Micrococcal nuclease (MN) (235 U/mg protein), apyrase, RNase A (type III-A) and RNase T1 (500,000 U/mg) were purchased from Sigma Chemical Co. (St. Louis, MO); nuclease P1 (389 U/mg dry weight) from Calbiochem (San Diego, $\mathrm{CA}$ ); proteinase $\mathrm{K}$ from Roche (Indianapolis, IN); spleen phosphodiesterase (SPD) $(2.45 \mathrm{U} / \mathrm{mg}$ ) from Worthington Biochemical Corp (Lakewood, NJ); and cloned T4 polynucleotide kinase $(10 \mathrm{U} / \mu \mathrm{l})$ from New England Bio Labs, Inc. (Beverley, MA). [ $\left.\gamma^{3}{ }^{32} \mathrm{P}\right] \mathrm{ATP}(6,000 \mathrm{Ci} / \mathrm{mmol})$ was purchased from NEN (Newton, MA) and Amersham (Piscataway, NJ). Saturated phenol, free from oxidant contaminants, was purchased from Amresco (Solon, $\mathrm{OH}$ ). Other chemicals and TLC solvents used for ${ }^{32} \mathrm{P}$-postlabeling were as described elsewhere (20).

Patients. All human samples were collected and analyzed after Institutional Review Board (IRB) approvals and ethics committee clearance from respective institutions. Brushings from normal $(n=13)$ and inflammatory cervix $(n=9)$ were obtained from subjects reporting for pap screening. Biopsies from severe dysplasias (CIN III) $(\mathrm{n}=5)$ and invasive cancers $(n=47)$ were obtained from patients at the gynecologic oncology/colposcopy unit. Of the cancer cases, 12 cases represented clinical stage IB; 22 cases represented stage II; and 13 cases represented stage III. Smears with no cytological abnormality were considered normal, and smears showing features of chronic cervicitis were classified as inflammatory cervix. Biopsies were classified based on histopathology reports as dysplasias and invasive cancers. The mean age
( \pm SD) and range of the patients in years in the normal, inflammation, dysplasia and cancer groups were 39 \pm (29-51),

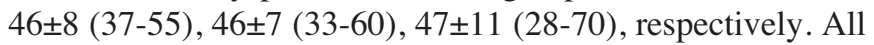
samples were collected at Cancer Institute, Chennai, India. Brushings were suspended in $10 \mathrm{ml}$ of phosphate-buffered saline, stored on ice and cells were pelleted within $1 / 2 \mathrm{~h}$ to $2 \mathrm{~h}$ of procurement and stored at $-80^{\circ} \mathrm{C}$ until isolation of DNA. Brushings and biopsied tissues were directly collected on ice from clinics and transferred immediately to $-80^{\circ} \mathrm{C}$ to avoid artefactual damage to DNA.

Isolation of DNA. DNA was isolated from the cervix samples by a solvent extraction procedure described in detail previously (20). Briefly, crude nuclei were isolated from frozen tissues or cells prior to digestion with RNAses and proteinase $\mathrm{K}$, followed by sequential extractions with phenol, phenol: sevag (chloroform:isoamyl alcohol, 24:1) and sevag. DNA was recovered by precipitation with ethanol in the presence of sodium chloride and dissolved in HPLC-grade water. The purity and concentration of DNA was determined spectrophotometrically. DNA was isolated at the Cancer Institute, Chennai, and transported to the US laboratory for analysis after the DNAs were sheared and lyophilized. Mean $( \pm$ SD) DNA recovery was $64 \pm 16 \mu \mathrm{g}$ from cytology specimens and $233 \pm 117 \mu \mathrm{g}$ from biopsies.

Analysis of DNA adducts by modified ${ }^{32} P$-postlabeling/TLC systems. DNA samples were analyzed by ${ }^{32} \mathrm{P}$-postlabeling method after enrichment of adducts with nuclease P1 (20), with modifications: briefly, DNA was digested with micrococcal nuclease and spleen phosphodiesterase (enzyme: substrate, 1:5) for $5 \mathrm{~h}$ at $37^{\circ} \mathrm{C}$. An aliquot was removed and used for evaluation of normal nucleotides and the remaining digest was treated with nuclease P1 (enzyme:substrate, $1: 2.5)$ for $1 \mathrm{~h}$ at $37^{\circ} \mathrm{C}$. Enriched digests were labeled with T4 polynucleotide kinase-catalyzed phosphorylation in the presence of $\left[\gamma_{-}{ }^{32} \mathrm{P}\right] \mathrm{ATP}(6,000 \mathrm{Ci} / \mathrm{mmol} ; 80 \mu \mathrm{Ci} ; 13.3 \mathrm{pmol})$. Labeled digests were resolved by 2-directional polyethyleneimine (PEI)-cellulose TLC $(20 \times 10 \mathrm{~cm})$ in $1 \mathrm{M}$ formic acid containing varying concentrations of sodium phosphate in direction 1 , followed by development in direction 2 in isopropyl alcohol:dilute ammonium hydroxide, as described in the Legend of Fig. 1. 8-OxodGp was enriched by PEI-cellulose TLC, ${ }^{32} \mathrm{P}$-labeled and resolved by 2 -directional TLC as described (21). Normal nucleotides were labeled in parallel with adducts and resolved by 1-directional PEI-cellulose TLC. Adduct and normal nucleotides were detected and quantitated by Packard InstantImager, and adduct levels were calculated as relative adduct labeling (RAL) as cpm in adduct(s)/cpm in normal nucleotides $\mathrm{x} 1 /$ dilution factor.

Statistical analysis. The analysis of data on DNA adducts was carried out in the context of Generalized Linear Models described by McCullagh (22). The data was analyzed using the Negative Binomial Regression model with logarithm as link function to study the dependence of the adduct levels on the type of subject (or the stage of subject). The parameters in the model were estimated using the method of maximum likelihood and the over-dispersion was accounted for by the scaled Pearson Chi-square. Statistical differences in adduct 
Table I. Levels of different subgroups of DNA adducts determined by ${ }^{32} \mathrm{P}$-postlabeling method.

\begin{tabular}{|c|c|c|c|c|c|c|c|c|}
\hline \multirow[b]{2}{*}{ Tissue } & \multicolumn{7}{|c|}{ Adducts $/ 10^{9}$ nucleotides } & \multirow[b]{2}{*}{ Total } \\
\hline & $\mathrm{P}-1$ & $\mathrm{P}-2 \mathrm{a}$ & P-2b (8-oxodG) & PL-1 & PL-2 & L-1 & L-2 & \\
\hline $\begin{array}{l}\text { Normal } \\
(\mathrm{n}=13)\end{array}$ & $1,343 \pm 432$ & $13,490 \pm 3,411$ & $1,886 \pm 507$ & $376 \pm 68$ & $70 \pm 22$ & $3,820 \pm 1,211$ & $144 \pm 61$ & $22,029 \pm 5,918$ \\
\hline $\begin{array}{l}\text { Inflammation } \\
(\mathrm{n}=9)\end{array}$ & $12,226 \pm 5,445$ & $10,708 \pm 3,209$ & $51,058 \pm 9,863$ & $2,380 \pm 436$ & $911 \pm 321$ & $11,902 \pm 2,073$ & $170 \pm 56$ & $89,684 \pm 13,755$ \\
\hline p-value & $<0.0001$ & 0.58 & $<0.0001$ & $<0.0001$ & $<0.0001$ & 0.0008 & 0.669 & $<0.0001$ \\
\hline $\begin{array}{l}\text { Dysplasia } \\
(\mathrm{n}=5)\end{array}$ & $12,369 \pm 9,413$ & $3,332 \pm 14,811$ & $30,362 \pm 10,354$ & $2,221 \pm 735$ & $458 \pm 388$ & $10,768 \pm 2,837$ & $424 \pm 152$ & $74,836 \pm 25,220$ \\
\hline p-value & $<0.0001$ & 0.9807 & $<0.0001$ & $<0.0001$ & 0.0137 & 0.0119 & 0.0224 & $<0.0001$ \\
\hline $\begin{array}{l}\text { Cancer stage I } \\
(\mathrm{n}=12)\end{array}$ & $4,471 \pm 785$ & $3,592 \pm 502$ & $12,371 \pm 4,184$ & $1,088 \pm 210$ & $328 \pm 62$ & $9,904 \pm 1,630$ & $263 \pm 47$ & $30,825 \pm 5,133$ \\
\hline $\begin{array}{l}\text { Cancer stage II } \\
(\mathrm{n}=22)\end{array}$ & $5,974 \pm 1,062$ & $4,436 \pm 1,024$ & $2,838 \pm 552$ & $1,789 \pm 215$ & $1,469 \pm 439$ & $17,132 \pm 2,445$ & $579 \pm 72$ & $35,318 \pm 3,386$ \\
\hline $\begin{array}{l}\text { Cancer stage III } \\
(\mathrm{n}=13)\end{array}$ & $9,351 \pm 2,769$ & $2,636 \pm 322$ & $3,916 \pm 436$ & $1,755 \pm 172$ & $580 \pm 61$ & $7,706 \pm 353$ & $595 \pm 117$ & $26,735 \pm 2,797$ \\
\hline $\begin{array}{l}\text { Cancer all stages } \\
(n=47)\end{array}$ & $6,681 \pm 977$ & $3,723 \pm 509$ & $5,659 \pm 1,371$ & $1,600 \pm 129$ & $932 \pm 218$ & $12,680 \pm 1,357$ & $503 \pm 51$ & $31,084 \pm 2,383$ \\
\hline p-value & $<0.0001$ & $<0.0001$ & 0.007 & $<0.0001$ & $<0.0001$ & $<0.0001$ & $<0.0001$ & 0.0349 \\
\hline
\end{tabular}

Values are represented as mean \pm SEM. P-values were calculated by comparison with normal group using Wald's asymptotic test procedures. A p-value of $<0.05$ was considered significant.

burden among different groups were calculated using Wald's asymptotic test procedures. A p-value of $<0.05$ was considered significant. The data analysis was carried out with the aid of SAS software version 8 .

\section{Results}

Over 60 adducts were detected in each DNA. Based on the elution profiles, six groups of adducts were detected which are designated as P-1, P-2, PL-1, PL-2, L-1 and L-2, where P stands for polarity and L for lipophilicity; the PL denotes adducts that are neither very polar nor lipophilic (Fig. 1). The adducts eluted in the following descending order of polarities: P-1, P-2, PL-1, PL-2, L-1 and L-2. The P-2 adducts were further classified as $\mathrm{P}-2$ a containing multiple adducts and $\mathrm{P}-2 \mathrm{~b}$ which represented a single entity, namely 8-oxodG, based on previous identification (21). With the exception of the P-2b adduct and L-2 adducts, all other groups of adducts detected have not been reported previously. Based on chromatographic mobility of $\left[\gamma^{32} \mathrm{P}\right] \mathrm{p} 8$-oxodGp, which resembled the mobility of the P-2a adducts, the 8 -oxodG adduct was coupled with P-2 adducts. Each group of adducts comprised of multiple adducts: at least 16 adducts in the P-1 subgroup, 11 adducts in the P-2a, and 3 and 4 adducts in the PL- 1 and PL-2 classes, respectively. The L-1 and L-2 adducts were poorly resolved or unresolved. In some analyses, however, the L-1 adducts separated in the form of beads of a string accounting for up to 25 adducts. A cluster of unresolved L-2 adducts migrated in the form of a diagonal radioactive zone (DRZ). This DRZ was embedded with at least five distinct adduct spots (Fig. 1). The L-2 adducts that are most lipophilic are detected with high-salt, high-urea solvents (23).

No qualitative differences were found in DNA adduct profiles from the four types of cervix specimens analyzed; i.e. normal, inflammation, dysplasia and invasive cancer. However, significant quantitative differences were found (Table I). The most pronounced adduct was 8-oxodG (P-2b) which represented $9 \%, 57 \%, 41 \%$ and $18 \%$ of the total adduct burden in the normal, inflammation, dysplasia and invasive cancer specimens, respectively. This adduct was elevated 27 -fold in cervix with inflammation $\left(51,058 \pm 9,863\right.$ adducts $/ 10^{9}$ nucleotides) compared with normal cervix $(1,886 \pm 507$ adducts/ $10^{9}$ nucleotides). This lesion was also significantly higher in the dysplasia $\left(30,362 \pm 10,354\right.$ adducts $/ 10^{9}$ nucleotides $)$ and invasive cancer $\left(5,659 \pm 1,371\right.$ adducts $/ 10^{9}$ nucleotides $)$. The $\mathrm{P}-1$ adducts in cervix with inflammation were higher by 9 -fold than that in normal cervix and remained at this level in the dysplasia group, but the increase was less pronounced (5-fold) in invasive cancer. The P-2a adduct levels in normal cervix did not differ from the inflammation and dysplasia groups. In fact, these adducts declined by approximately $70 \%$ in invasive cancer. The PL-1 and PL-2 adducts in cervix with inflammation were also elevated, by 6- and 13-fold, respectively, and these levels remained high through dysplasia and invasive cancer conditions. The L-1 adducts in the inflammation group were higher by 3 -fold and remained practically constant through 
A

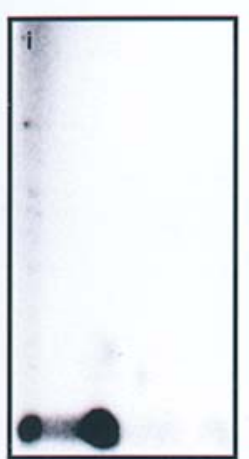

- DNA

B

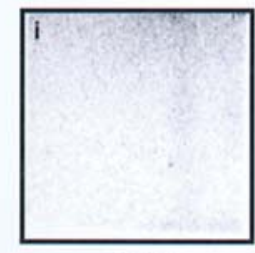

- DNA

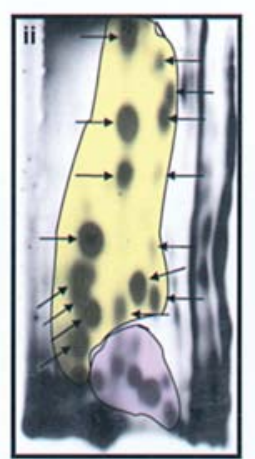

$\mathrm{P}-1 / \mathrm{P}-2 \mathrm{a}$

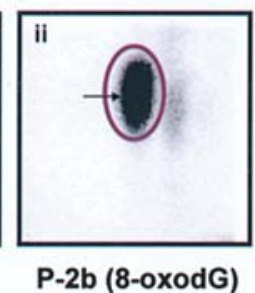

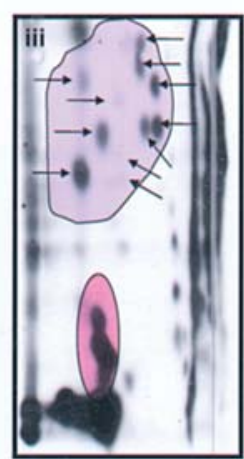

P-2a/PL-1

C

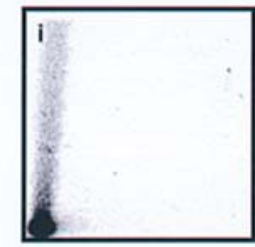

- DNA

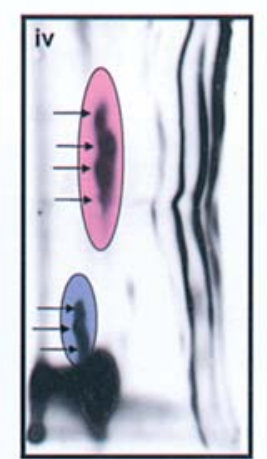

PL-1/PL-2

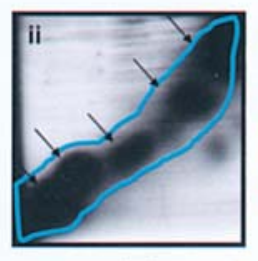

L-2

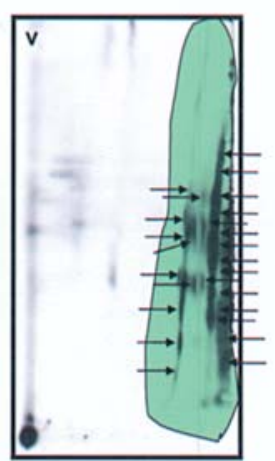

L-1

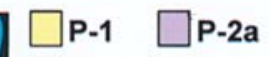

$\square$ P-2b $\square$ PL-1 $\square \mathrm{PL}-2 \square \mathrm{L}-1$

L-2

Figure 1. Representative maps of ${ }^{32} \mathrm{P}$-adducts from human cervix DNA isolated from Pap smears were analyzed by ${ }^{32} \mathrm{P}$-postlabeling in conjunction with PEIcellulose TLC. Following enzymatic digestion of DNA, adducted nucleotides were enriched by treatment with nuclease P1, except for 8-oxodGp which was enriched by PEI-cellulose TLC (21). Adducts were labeled and chromatographed by 2- or 3-directional PEI-cellulose TLC. The nuclease P1-enriched, newly discovered, array of adducts were resolved on four sheets $(10 \times 20 \mathrm{~cm}$; each $8 \mu \mathrm{g})$ by development in $1 \mathrm{M}$ formic acid containing increasing salt concentrations: $50 \mathrm{mM}$ (A-ii), $100 \mathrm{mM}$ (A-iii), $150 \mathrm{mM}$ (A-iv) and $240 \mathrm{mM}$ (A-v) sodium phosphate, pH 6.0 (direction 1; from bottom to top). After removal of the salt by washing in water, followed by drying, the sheets were developed in 1:1 isopropanol:4 $\mathrm{N}$ ammonium hydroxide (direction 2 , from left to right). The highly lipophilic (L-2) adducts were resolved on 10x13-cm sheets by development in $1 \mathrm{M}$ sodium phosphate, $\mathrm{pH} 6.0$ (direction 1, bottom to top), $3 \mathrm{M}$ lithium formate, $7 \mathrm{M}$ urea, pH 3.5 (direction 2, bottom to top) and 0.8 M lithium chloride, 0.5 M Tris-HCl, $7 \mathrm{M}$ urea, pH 8.0 (direction 3, left to right) (C-ii). 8-OxodG adducts were resolved in $0.6 \mathrm{M}$ formic acid (direction 1, from bottom to top) and $3.0 \mathrm{M}$ sodium phosphate, pH 6.0 (direction 2 , left to right). The development in direction 1 was onto a $6-\mathrm{cm}$ Whatman \#17 wick attached to the top of the sheet, and development in directions 2 and 3 were to the top of the sheet ( B-ii). A-i, $\mathrm{B}-\mathrm{i}$ and $\mathrm{C}$-i were processed as controls in parallel with A-ii, B-ii and C-ii, respectively by omitting DNA. Adduct spots were detected by Kodak X-Omat film exposure $\left(-80^{\circ} \mathrm{C}\right.$, for 6-24 h). For quantitation, adducts were visualized by Packard InstantImager.

dysplasia and invasive cancer. The levels of the highly lipophilic L-2 adducts, in the normal and inflammation groups were not different, but the levels then increased steadily, reaching 3-fold higher in the invasive cancer. When comparing total adduct burden, the levels in both inflammation and dysplasia groups were significantly higher than in normal cervix. In the invasive cancer, the total adduct burden was also higher than in normal cervix but the increase was statistically insignificant. It may be noted that the burden of some subgroups of adducts in the inflammation, dysplasia and invasive cancer were much more elevated than the total adduct burden (Table I).

The modulation of individual adducts in all subgroups largely reflected the modulation of total adducts. Therefore, we have described the modulation of total adducts under each subgroup. To determine if adduct burden varied during the invasiveness of the cancer, we compared the adduct profiles from stage I versus stages II and III. Again, no qualitative differences were noticed, but significant quantitative differences were found. For instance, the 8-oxodG levels declined to one third from stage I to stage II and then no further decline occurred, but the levels of PL-1, PL-2, L-1 and L-2 all increased by 2- to 5-fold from stage I to stage II and then in stage III the adduct levels either remained unchanged or declined (Table I).

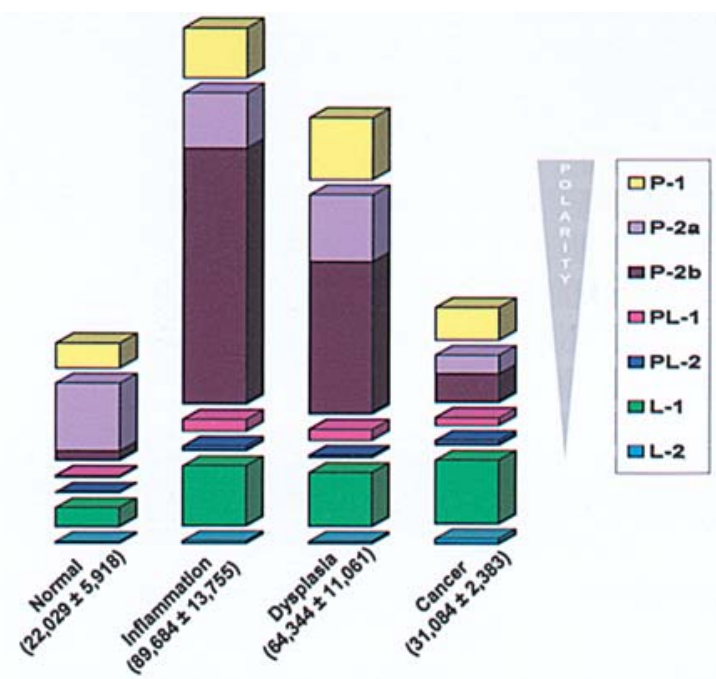

Figure 2. Alterations in DNA adduct profiles in different stages of cervical pathology represented by stacking the levels of each subgroup of adducts. Values represent adducts $/ 10^{9}$ nucleotides (mean \pm SEM).

Data presented in Table I also reveal that the previously known lipophilic adducts (the L-2 adducts) represent only a very small fraction $(0.1-0.5 \%)$ of the total adduct burden. 

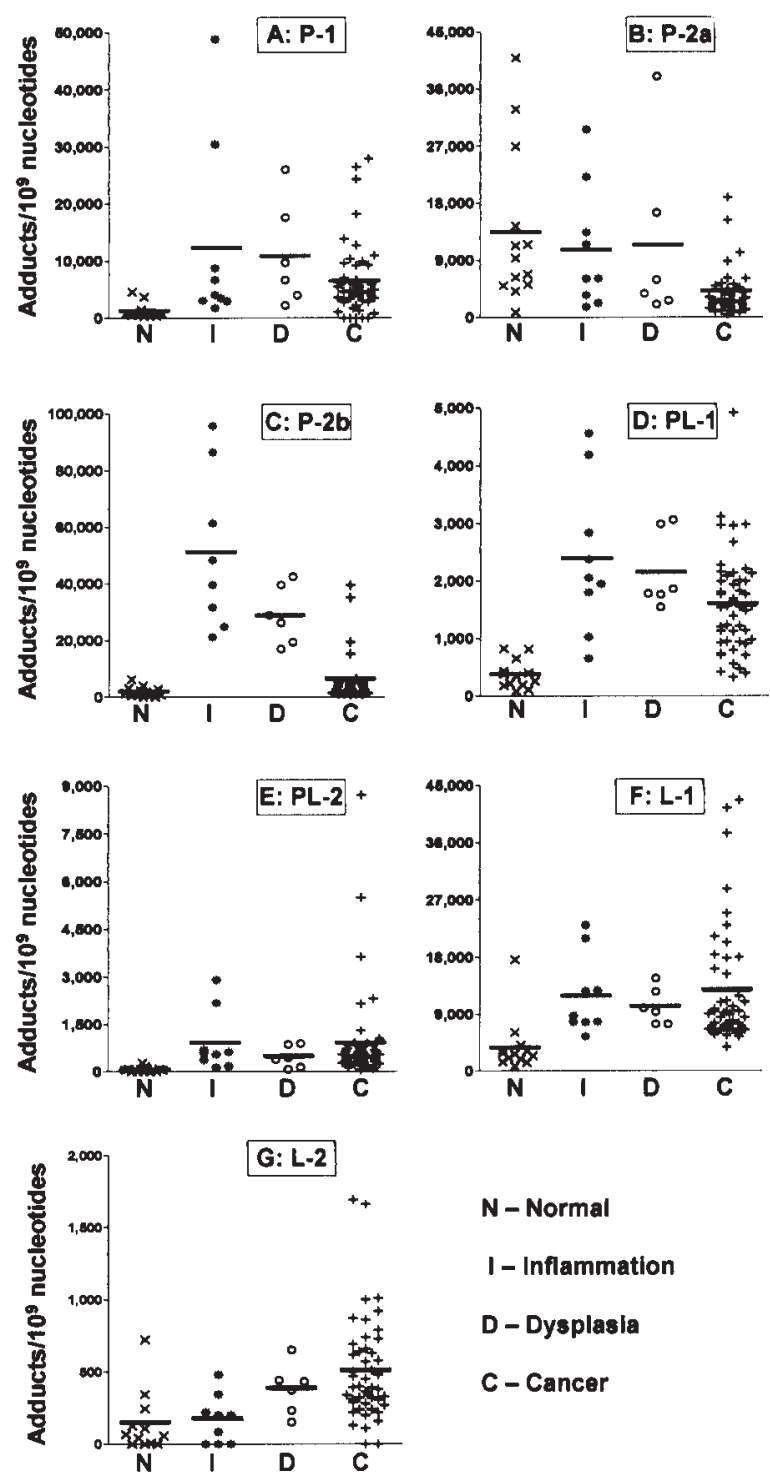

Figure 3. Scatter plots highlighting the inter-individual variability of different subgroups of adducts in various cervical pathology.

The changes in adduct profiles that occur during the progression of cervical cancer are represented, for simplicity, by stacking the levels of each group of adducts (Fig. 2). Clearly, adducts, particularly in the P-1, P-2b and L-1 groups, accumulate the most in cervix with inflammation.

The interindividual differences observed in the levels of each group of adducts varied depending upon the diseased state of the cervix, as illustrated by the scatter plots (Fig. 3). While there was a 3- to 5-fold variability in the total adduct burden in inflammation, dysplasia and invasive cancer groups, the variability was up to 27 -fold in the normal individuals. This could be indicative of the susceptibility of some of these individuals to accumulate more damage and warrants a longitudinal study to better understand the biomarker potential of this newly identified group of adducts.

We also tested 5 residual negative cytology specimens obtained from the University of Kentucky Hospital, and found that the DNA recovery (mean value $=70 \pm 39 \mu \mathrm{g}$ ) and adduct profiles (data not shown) were essentially the same as in the Chennai samples.

\section{Discussion}

Techniques used to detect and identify endogenous DNA adducts include immunoassay, gas chromatography-mass spectrometry, liquid chromatography-mass spectrometry and ${ }^{32} \mathrm{P}$-postlabeling. The former three techniques have been valuable in identifying many endogenous DNA adducts, however, they either apply to targeted adducts (immunoassay), or they require relatively large quantities of DNA $(100-500 \mu \mathrm{g})$ (spectrometric techniques). The ${ }^{32} \mathrm{P}$-postlabeling assay, a standard in the field for adducts from exogenous carcinogens, requires relatively small quantities of DNA $(<10 \mu \mathrm{g})$ and can detect $<1$ adduct in $10^{9}$ nucleotides. It, however, does not allow adduct identification without authentic standards. Only a few known oxidative adducts have been measured by this assay. No attempt has been made so far to detect the numerous structurally diverse endogenous DNA adducts in tissues during the progression of human cancer.

With the exception of 8-oxodG (P-2b), all other adducts remain as yet unidentified. It may be noted that the newly discovered array of adducts were present at 61- to 527-fold higher levels than the previously reported highly lipophilic (L-2) adducts (Table I). Interestingly, data indicating that 8oxodG levels in cervical cancer are 3-fold higher compared with normal cervix, and that cervix with inflammation has dramatically (27-fold) elevated levels of this oxidative lesion than normal cervix have not been reported previously.

A causal relationship between inflammation and cancer was speculated as early as 1863 by Rudolph Virchow (cited in ref. 24). Development of several cancers has been attributed to chronic inflammatory diseases (reviewed in ref. 25). Lately, the association between inflammation and cancer is receiving greater attention (26-29). We found a parallel increase of the inflammatory cytokines IL-6 and IL-8, neutrophils and macrophages and several novel adducts, suggesting a possibility of inter-relationship (unpublished data). Rapidly dividing cells are most prone to errors in DNA replication and repair. Besides, hot spots for damage could more often be cold spots for repair (30). The distribution of many polar adducts discovered in this study may influence the disease status as it progresses from normal cervix through inflammation by accruing mutations.

Several studies have shown an association of smoking with the known highly lipophilic group of adducts (the DRZ) in the cervix (31-33). But there are no studies that have analyzed DNA adducts at different stages as the disease progresses to malignancy. Smoking was found to increase the risk of cervical cancer among HPV-positive women in a multicentric case-control study (32). Benzo[a]pyrene diolepoxideDNA adducts were reported in the cervical epithelium of smokers and the role of polycyclic aromatic hydrocarbons and their carcinogenic metabolites in cervical cancer has been described by Melikian et al (33). A progressive increase in the accumulation of adducts that migrate in the form of DRZ on the chromatograms suggests a positive role of these modifications in cervical carcinogenesis. Although passive smoking and tobacco chewing is widespread, smoking is not a common practice among women in the region where these samples were collected. It is therefore quite possible that the 


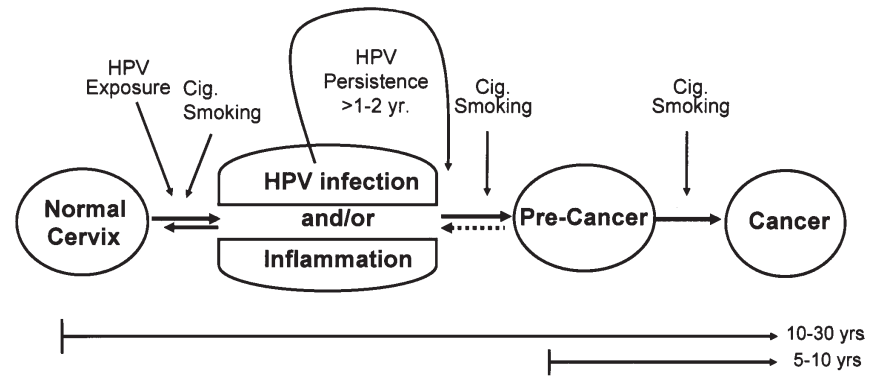

Figure 4. Schematic representation of the proposed hypothesis on the role of co-factors in cervical carcinogenesis. Adapted and modified from Wright and Schiffman (39).

DRZ adducts could also accumulate due to factors other than smoke constituents.

The differential accumulation of different groups of adducts suggest that while L-2 adducts continue to form throughout the disease progression, the PL- 1 and L-1 adducts may form at higher rates than the rate of removal initially and thereafter the rates of formation and repair may parallel. A possible explanation for the decline of adducts in cancerous tissues could result, in part, from dilution with newly replicated DNA. The malignant cells undergo high turnover and do not stay in the resting or G0 phase for long unlike normal cells. Adducts may be diluted in this process as they do not have enough time to accumulate. Nevertheless, this high cell turnover may promote fixation of these lesions leading to initiation of the process of tumorigenesis.

The levels of P-2a adducts are lower in cancer tissues compared with normal cervix or cervix with inflammation and dysplasia. It remains to be determined if these adducts may represent some kind of essential modifications required for the normal functioning of cellular activities. One such example is 5-methylcytosine in DNA. Certain regions of the genome defined as $\mathrm{CpG}$ islands contain a predicted frequency of $\mathrm{CpG}$ dinucleotides that are unusually unmethylated, except for those on the inactive $\mathrm{X}$ chromosome and parentally imprinted genes (34). Global hypomethylation has been implicated in oncogenic transformations $(35,36)$.

Data from Table I clearly indicate a significant increase in the various adduct groups from normal to cancer except P-2a where a significant decrease was observed. This skews the total adduct level and hence a statistically significant change was not observed. It may therefore not be appropriate to compare the total adduct burden.

The profiles of newly detected adducts are qualitatively similar to the patterns observed in several other organs in normal and disease status in humans (unpublished data). The similarity in adduct profiles in the Chennai cervix samples and the Kentucky cervix samples suggest that these adducts may be derived from endogenous factors and may not be specific to any ethnic group or environmental factors. This exemplifies the qualitative similarity and quantitative differences in the levels of these new sets of adducts in different cervix pathology, and their implications in the progression of cancer. The new group of adducts also showed substantial inter-individual variability. Analysis of the individual adduct levels shows a heterogeneous pattern of adduct distribution as shown by the scatter plots (Fig. 3), suggestive of susceptibility differences among individuals in accumulating and repairing these lesions. A possible explanation may be the difference in their repair capacity due to polymorphisms in select DNA repair genes. This inter-individual variability may facilitate the identification of high-risk populations and planning of intervention strategies.

Our findings support the hypothesis that infections of the cervix lead to inflammation, and the release of ROS by endogenous factors leads to high oxidative damage to the DNA. Some of the modifications thus accumulated may lead to irreversible changes in the genome and initiate cancer. Several studies have linked smoking and cervical carcinogenesis. Possible mechanisms of smoking-related cervical carcinogenesis include suppression of DNA repair capacity (37) leading to accumulation of DNA damage and induction of further DNA damage by the smoke constituents (reviewed in ref. 38). We therefore propose a scheme for cervix carcinogenesis (Fig. 4). HPV infection is assumed to be a necessary but insufficient step for carcinoma development. Many, if not the majority of women, are exposed to HPV at a young age, but only a few women develop cancer. Persistent infection (>1-2 years) with a high-risk HPV type increases the risk status of a woman. Our focus in this scheme is on the role of inflammation and associated factors like cigarette smoking, that promote the progression of a HPV-infected cervix to cancer. Since there are no appropriate markers with which to monitor disease during progression, high-grade intraepithelial lesions are often treated by a surgical resection or loop excision, which may be an over-treatment for several patients. Identification of biomarkers that predict tumor formation and progression would improve drastically the current clinical scenario. Although these novel adducts detected are indicative of an association between inflammation and cancer, it may be noted that this is a cross-sectional study. Further evaluation of these adducts that show a significant quantitative change in disease progression in a longitudinal study may prove them to be suitable markers of risk progression for the identification of high-risk subjects.

\section{Acknowledgements}

This work was supported from the USPHS grant CA-77114, and in part from Agnes Brown Duggan Endowment Funds.

\section{References}

1. Marnett LJ: Oxyradicals and DNA damage. Carcinogenesis 21: 361-370, 2000.

2. Jaiswal M, La Russo NF, Burgart LJ and Gores GJ: Inflammatory cytokines induce DNA damage and inhibit DNA repair in cholangiocarcinoma cells by a nitric oxide-dependent mechanism. Cancer Res 60: 184-190, 2000.

3. Salvemini D and Cuzzocrea S: Superoxide, superoxide dismutase and ischemic injury. Curr Opin Investig Drugs 3: 886-895, 2002.

4. Halliwell B and Aruoma OI: DNA damage by oxygen-derived species. Its mechanism and measurement in mammalian systems. FEBS Lett 281: 9-19, 1991.

5. Gupta RC and Lutz WK: Background DNA damage for endogenous and unavoidable exogenous carcinogens: a basis for spontaneous cancer incidence? Mutat Res 424: 1-8, 1999.

6. Marnett LJ and Burcham PC: Endogenous DNA adducts: potential and paradox. Chem Res Toxicol 6: 771-785, 1993.

7. Nair J, Barbin A, Velic I and Bartsch H: Etheno DNA-base adducts from endogenous reactive species. Mutat Res 424: 59-69, 1999. 
8. Chung FL, Nath RG, Nagao M, Nishikawa A, Zhou GD and Randerath K: Endogenous formation and significance of 1,N2-propanodeoxyguanosine adducts. Mutat Res 424: 71-81, 1999.

9. Marnett LJ: Chemistry and biology of DNA damage by malondialdehyde. IARC Sci Publ 150, pp17-27, 1999.

10. Follen M and Schottenfeld D: Surrogate endpoint biomarkers and their modulation in cervical chemoprevention trials. Cancer 91: 1758-1776, 2001

11. Jemal A, Siegel R, Ward E, et al: Cancer statistics, 2006. CA Cancer J Clin 56: 106-130, 2006.

12. Wyatt SW, Lancaster M, Bottorff D and Ross F: History of tobacco use among Kentucky women diagnosed with invasive cervical cancer: 1997-1998. J Ky Med Assoc 99: 537-539, 2001.

13. Walboomers JM, Jacobs MV, Manos MM, et al: Human papillomavirus is a necessary cause of invasive cervical cancer worldwide. J Pathol 189: 12-19, 1999.

14. Ostor AG: Natural history of cervical intraepithelial neoplasia: a critical review. Int J Gynecol Pathol 12: 186-192, 1993.

15. Castellsague X, Bosch FX and Munoz N: Environmental cofactors in HPV carcinogenesis. Virus Res 89: 191-199, 2002.

16. Castle PE, Hillier SL, Rabe LK, et al: An association of cervical inflammation with high-grade cervical neoplasia in women infected with oncogenic human papillomavirus (HPV). Cancer Epidemiol Biomarkers Prev 10: 1021-1027, 2001.

17. Tjiong MY, van der Vange N, ten Kate FJ, et al: Increased IL-6 and IL-8 levels in cervicovaginal secretions of patients with cervical cancer. Gynecol Oncol 73: 285-291, 1999.

18. Srivani $\mathrm{R}$ and Nagarajan B: A prognostic insight on in vivo expression of interleukin-6 in uterine cervical cancer. Int J Gynecol Cancer 13: 331-339, 2003.

19. Srivani R, Pratibha K, Selvaluxmy G and Nagarajan B: Molecular logistics of cytokines in cervical cancer. Curr Sci 84: 434-438, 2003

20. Gupta RC: ${ }^{32}$ P-Postlabeling for detection of DNA adducts. In: Technologies for detection of DNA damage and mutations. Pfeifer GP (ed.) Plenum Press, New York, pp45-61, 1996.

21. Gupta RC and Arif JM: An improved ( $\left.{ }^{32}\right) \mathrm{P}$-postlabeling assay for the sensitive detection of 8-oxodeoxyguanosine in tissue DNA. Chem Res Toxicol 14: 951-957, 2001.

22. McCullagh P and Nelder JA: Generalized Linear Models. Chapman \& Hall Inc., 1989.

23. Phillips DH, Schoket B, Hewer A and Grover PL: Human DNA adducts due to smoking and other exposures to carcinogens. Prog Clin Biol Res 340C: 283-292, 1990.
24. Balkwill $F$ and Mantovani A: Inflammation and cancer: back to Virchow? Lancet 357: 539-545, 2001.

25. Coussens LM and Werb Z: Inflammation and cancer. Nature 420: 860-867, 2002

26. Mantovani A: Cancer: inflammation by remote control. Nature 435: 752-753, 2005

27. De Visser KE, Korets LV and Coussens LM: De novo carcinogenesis promoted by chronic inflammation is B lymphocyte dependent. Cancer Cell 7: 411-423, 2005.

28. Zavros Y, Eaton KA, Kang W, et al: Chronic gastritis in the hypochlorhydric gastrin-deficient mouse progresses to adenocarcinoma. Oncogene 24: 2354-2366, 2005.

29. Nickoloff BJ, Ben-Neriah Y and Pikarsky E: Inflammation and cancer: is the link as simple as we think? J Invest Dermatol 124: x-xiv, 2005.

30. Singer B and Hang B: What structural features determine repair enzyme specificity and mechanism in chemically modified DNA? Chem Res Toxicol 10: 713-732, 1997.

31. Phillips DH and She MN: DNA adducts in cervical tissue of smokers and non-smokers. Mutat Res 313: 277-284, 1994.

32. Plummer M, Herrero R, Franceschi S, et al: Smoking and cervical cancer: pooled analysis of the IARC multi-centric case control study. Cancer Causes Control 14: 805-814, 2003.

33. Melikian AA, Sun P, Prokopczyk B, et al: Identification of benzo[ $[a]$ pyrene metabolites in cervical mucus and DNA adducts in cervical tissues in humans by gas chromatography-mass spectrometry. Cancer Lett 146: 127-134, 1999.

34. Li E, Beard C and Jaenisch R: Role for DNA methylation in genomic imprinting. Nature 366: 362-365, 1993.

35. Das PM and Singal R: DNA methylation and cancer. J Clin Oncol 22: 4632-4642, 2004.

36. Davis CD and Uthus EO: DNA methylation, cancer susceptibility, and nutrient interactions. Exp Biol Med 229: 988-995, 2004.

37. Thun MJ, Henley SJ and Calle EE: Tobacco use and cancer: an epidemiologic perspective for geneticists. Oncogene 21: 7307-7325, 2002.

38. Phillips DH: Smoking-related DNA and protein adducts in human tissues. Carcinogenesis 23: 1979-2004, 2002.

39. Wright TC Jr and Schiffman M: Adding a test for human papillomavirus DNA to cervical-cancer screening. N Engl J Med 348: 489-490, 2003 